\title{
POLÍTICA NACIONAL Y VISIBILIDAD INTERNACIONAL. EL CASO COLOMBIANO
}

Silvia Molina-Molina y Félix De-Moya-Anegón

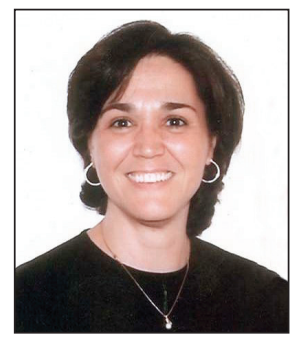

Silvia Molina-Molina es bibliotecóloga por la Escuela Interamericana de Bibliotecología de la Universidad de Antioquia (UdeA), donde actualmente se desempeña como profesora e integrante del Grupo de Investigación en Información, Conocimiento y Sociedad, en razón del cual adelanta los proyectos institucionales Observatorio de la Investigación y Evaluación de Revistas Científicas. Es doctora en información científica por la Universidad de Granada y candidata a integrante junior de SCImago Research Group. http://orcid.org/0000-0003-4482-7483

Grupo de investigación en Información, Conocimiento y Sociedad, Escuela Interamericana de Bibliotecología Universidad de Antioquia (UdeA) Calle 70, No. 52-21, Medellín, Colombia SCImago Research Group, msmoli@bibliotecologia.udea.edu.co

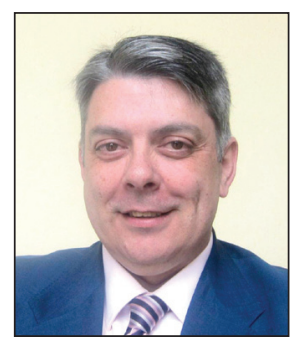

Félix De-Moya-Anegón, doctor en historia por la Universidad de Granada, es profesor de investigación en el Instituto de Políticas y Bienes Públicos del Consejo Superior de Investigaciones Científicas (CSIC) en Madrid e investigador principal de la unidad asociada SCImago Research Group que ha llevado a cabo los proyectos de I+D SCImago Journal \& Country Rank (SJR), SCImago Institutions Rankings (SIR), Atlas de la Ciencia, etc. Su última publicación web es el Ranking Iberoamericano SIR 2013.

http://orcid.org/0000-0002-0255-8628

Centro de Ciencias Humanas y Sociales (CCHS), CSIC Albasanz, 26-28, 28037 Madrid, España SCImago Research Group felix.moya@scimago.es

\section{Resumen}

Se analizan los efectos positivos y negativos de las políticas colombianas dirigidas a incentivar la producción científica de los profesores universitarios, además de aquellas que rigen para las instituciones de educación superior dentro de los procesos de acreditación de la calidad y la asignación de recursos del presupuesto nacional. Se pone en evidencia que si bien Colombia reporta la mayor tasa de crecimiento de los países latinoamericanos en la base de datos Scopus y está mejorando sus prácticas de comunicación científica, tiene el reto de acompañar los resultados de este desempeño, con un mayor énfasis en los indicadores de calidad. Son necesarios ajustes a las políticas relacionadas con la producción científica colombiana en aras de una mayor visibilidad internacional y un sistema de incentivos a investigadores e instituciones de educación superior más eficiente. En esta dirección empiezan a orientarse las nuevas disposiciones.

\section{Palabras clave}

Política de información, Política científica, Política nacional, Revistas científicas, Visibilidad internacional, Publindex, Colombia.

Title: National policy and international visibility. The Colombian case

\section{Abstract}

The positive and negative effects of policies aimed at encouraging scientific output by university professors in Colombia, in addition to those that apply to higher education institutions in the quality accreditation process and in national budget resource allocation, are discussed. Although Colombia reported the highest rate of growth among Latin American countries in the Scopus database and is improving its scientific communication practices, the country now has the challenge of combining these performance results with a greater emphasis on quality indicators. In order to achieve greater international visibility, policy adjustments related to improving scientific production are needed, as well as a more efficient incentives system for researchers and higher education institutions. New policy provisions are beginning to be focussed in this direction.

\section{Keywords}

Information policy, Science policy, National policy, Scientific publications, International visibility, Publindex, Colombia. 
Molina-Molina, Silvia; De-Moya-Anegón, Félix (2013). “Política nacional y visibilidad internacional. El caso colombiano". El profesional de la información, noviembre-diciembre, v. 22, n. 6, pp. 529-535.

http://dx.doi.org/10.3145/epi.2013.nov.05

\section{Introducción}

La producción científica colombiana registrada en la base de datos Scopus reporta la mayor tasa de crecimiento de los países latinoamericanos. Este comportamiento se explica parcialmente por el ingreso de revistas nacionales en esta base de datos, como resultado de la política de Elsevier de ampliar la cobertura geográfica de Scopus, y así diferenciarse, entre otras características, de la base de datos ISI Web of Science, con quien entró a competir desde 2004 en el mercado de los índices de citaciones internacionales y multidisciplinares. Esto le ha permitido a Colombia tener 59 revistas científicas nacionales indexadas en 2012, cuando hace apenas pocos años sólo se registraba un título en la base de datos ISI Web of Science.

Este proceso se ha llevado a cabo gradualmente, conforme Scopus amplía su cobertura por regiones; pero también, en la medida en que Colombia gana madurez científica y avanza en sus políticas de ciencia, tecnología e innovación, las cuales acompaña de estrategias de evaluación de la ciencia e incentivos a la producción científica. Entre estas estrategias se pueden mencionar cinco que están directamente relacionadas con la comunicación científica:

- el régimen salarial de los profesores de las universidades públicas;

- la clasificación de revistas científicas nacionales; el modelo de medición de grupos de investigación;

- la asignación de recursos a las universidades estatales; y

- el sistema de acreditación de la calidad de instituciones y programas de pregrado y posgrado.

\section{Estrategias colombianas}

\subsection{Régimen salarial de los profesores universitarios}

La actual Constitución Política de Colombia data de 1991 y a partir de ella se define una nueva época para la realidad universitaria, que empieza por organizar el Sistema de Educación Superior mediante la Ley 30 de 1992 (Colombia, 1992a) para luego promulgar el Decreto 1444 de 1992 (Colombia, $1992 b)$ que establece el régimen salarial de los profesores de las universidades públicas del orden nacional asociándolo con la productividad académica, entre otros aspectos. Así, el Decreto establece una escala de puntajes para reconocer los trabajos publicados según tipos de productos, previa condición para los profesores de "acreditar su vinculación con la universidad respectiva y dar crédito o mención a ella". En el caso de las publicaciones en revistas, se establecen tres categorías: revistas especializadas del exterior de nivel internacional; revistas especializadas nacionales de nivel internacional; y revistas especializadas nacionales de circulación nacional o regional. Estas categorías se mantienen hasta el 2001, cuando se promulga el Decreto 2912 (Colombia, 2001) que deroga el 1444 y acoge el Sistema Nacional de Indexación y Homologación de Revistas Especiali- zadas en $C T+1$, creado por Colciencias (la agencia estatal que promueve las políticas públicas para el fomento de la $\mathrm{CT}+\mathrm{I}$ en Colombia), en colaboración con el Observatorio Colombiano de Ciencia y Tecnología. Actualmente rige el Decreto 1279 de 2002 (Colombia, 2002), que asimismo se guía por ese Sistema; no obstante, también está siendo sometido a revisión como uno de los puntos críticos de la desfinanciación de la educación superior, pues se considera que estos decretos "introdujeron un modelo de estímulos salariales y bonificaciones por formación, experiencia y productividad académica que no tiene límite de crecimiento, es decir, no tiene tope máximo de puntos acumulados a obtener por docente y por ende su salario puede crecer ilimitadamente" (Colombia. Sistema Universitario Estatal, 2012, p. 48).

Estos Decretos también determinan la cultura de la comunicación científica de Colombia, siendo especialmente adverso el numeral III que establece "restricciones de puntajes según el número de autores" y disminuye los puntajes que se asignan conforme se eleva el número de autores por artículo. De esta manera, se limita la colaboración científica que tanto favorece la visibilidad.

\subsection{Clasificación de las revistas científicas nacionales}

El Sistema Nacional de Indexación y Homologación de Revistas Especializadas en $\mathrm{CT}+\mathrm{l}$ incluye la Base Bibliográfica $\mathrm{Na}$ cional, que contiene la información de las revistas científicas y académicas nacionales hasta el nivel de artículos a texto completo y también los títulos de las revistas extranjeras homologadas. Para el efecto, se llevan a cabo sendos servicios: el servicio permanente de indexación y el servicio de homologación (Colombia. Colciencias, 2013a).

El servicio permanente de indexación da lugar al Índice Bibliográfico Nacional - IBN Publindex, que se lleva a cabo conforme a criterios establecidos en un documento que se divulga entre los editores como guía del proceso de indexación y que tiene varias versiones, según se van consolidando los criterios y se ajustan a parámetros internacionales. El más reciente data de 2013 (Colombia. Colciencias, 2013b). La actualización del índice Publindex se realiza dos veces al año, previa convocatoria y análisis de la información suministrada por los editores en una plataforma informática que permite la actualización online de la información de las revistas y de sus contenidos.

El servicio de homologación está diseñado para identificar y reconocer las revistas extranjeras especializadas de $\mathrm{CT}+\mathrm{I}$ en una de las categorías establecidas para el Sistema Nacional de Indexación y Homologación. El listado de estas revistas se actualiza durante el segundo semestre de cada año, luego que los servicios de indexación y resumen seleccionados por Publindex han publicado sus respectivas actualizaciones.

Tanto el servicio de indexación como el de homologación, tienen en cuenta los lineamientos trazados respecto a los 
servicios de indexación y resumen (sires) considerados de calidad, así como el documento guía ya mencionado. De ellos se tienen algunas versiones que se van ajustando en cada convocatoria, siendo las más recientes las publicadas en 2013 que introducen cambios importantes en los criterios (Colombia. Colciencias, 2013c). Uno de ellos intenta corregir una falla estructural del sistema, según la cual los índices de citas Science Citation Index, Social Science Citation Index y Scopus no tenían una valoración destacada entre los servicios de indización y resúmenes seleccionados por Colciencias. A partir de 2013, sí se establecen claramente las diferencias y se clasifican en la máxima categoría, diferenciando además, la ubicación en los cuartiles. En el proceso de medición de grupos la lista de sires se amplía de 26 a 83 (Colombia. Colciencias, 2013c), pero todavía es un documento en proceso de aplicación y luego habrá que analizar los resultados de este cambio.

Con ese Sistema, Colciencias busca "incrementar y fortalecer la calidad, cantidad, visibilidad, impacto y reconocimiento de las revistas nacionales especializadas de ciencia, tecnología e innovación, así como los artículos científicos publicados en ellas". Adicionalmente, lleva a cabo un programa de capacitación para editores y de promoción de la cultura Open Access para las mismas revistas científicas nacionales, así como su inclusión en bases de datos de acceso abierto y en repositorios (Colombia. Colciencias, 2013a).

Charum (2004) analiza detalladamente la historia de Publindex, siendo ese autor uno de sus protagonistas. Por su parte, Delgado-Troncoso (2009) también presenta las características de este índice y su papel en la evolución de las capacidades de ciencia, tecnología e innovación de Colombia.

\subsection{Modelo de medición de grupos de investigación}

Contrario a los sistemas nacionales de ciencia, tecnología e innovación del mundo, el colombiano centra su atención en los grupos de investigación más que en los investigadores, y es en función de ellos que se evalúa la actividad científica del país. A finales de los años noventa se inicia la construcción de un escalafón para categorizar los grupos y en 2002 se lanza en Colombia la plataforma ScienTI que reúne, entre otras, las bases de datos CVLAC y GrupLAC, con los curriculum vitae de investigadores de América Latina y El Caribe, y brindan información sobre los grupos de investigación de la misma región, respectivamente.

http://www.colciencias.gov.co/web/guest/scientihome

Ellas constituyen las principales fuentes de consulta sobre la producción científica que declaran y registran online los mismos investigadores y coordinadores de grupo.

Colciencias realiza convocatorias nacionales para la medición de grupos de investigación, tecnología e innovación, mediante las cuales los invita a concursar para su registro o reconocimiento, según un modelo y marco conceptual predefinido y divulgado ampliamente (Colombia. Colciencias, 2013d). En todos los casos, el modelo ha establecido pesos específicos para la producción académica que está en total correspondencia con el sistema Publindex. De hecho, para la versión de 2013 se trabajaron en simultánea los documen- tos referentes a la medición de grupos, la indexación de revistas y los sires, como se ha descrito.

\subsection{Asignación de recursos a las universidades esta- tales}

El Sistema de Universidades del Estado (SUE) también se crea con la Ley 30 de 1992 y tiene entre sus objetivos "crear las condiciones para la realización de evaluación en las instituciones pertenecientes al sistema". De esta evaluación depende la distribución de recursos que anualmente hace el Ministerio de Educación Nacional entre las universidades estatales u oficiales. Igual que sucede con los demás modelos de medición mencionados, el del SUE ha experimentado varias modificaciones. A partir de 2004 se logra definir la batería de indicadores que sirven como base para la asignación de los recursos, pero sólo logra estabilizarse en el año 2010, permitiendo que los indicadores utilizados en 2011 y 2012 sean casi los mismos (Colombia. Ministerio de Educación Nacional, 2012). Se trata de 31 indicadores que dan cuenta de resultados agregados y de capacidad del SUE. Respecto a la medición de la ciencia, el Índice de investigación (Índice de resultados de producción científica, Irprod) evalúa lo siguiente:

- Número ponderado de grupos de investigación reconocidos y escalafonados por Colciencias.

- Número ponderado de revistas indexadas de la institución de acuerdo con la legislación vigente (Colciencias).

- Número ponderado de artículos de carácter científico, técnico, artístico, humanístico o pedagógico publicados en revistas indexadas.

- Número de patentes nacionales e internacionales y secretos industriales de la institución.

- Movilidad de docentes e investigadores de instituciones de educación superior (IES) colombianas en el exterior.

- Número de productos audiovisuales, cinematográficos o fonográficos y obras artísticas.

- Empresas de innovación y/o base tecnológica creadas a partir de la investigación (spin offs).

(Colombia. Ministerio de Educación Nacional, 2012).

\subsection{Sistema de acreditación de la calidad}

El Consejo Nacional de Acreditación (CNA), por su parte, evalúa la calidad de los programas académicos de las instituciones de educación superior (Colombia. Consejo Nacional de Acreditación, 2010) y a las instituciones como un todo (Colombia. Consejo Nacional de Acreditación, 2006). Incluye indicadores relacionados con el número de artículos publicados por los profesores cada año, en revistas internacionales indexadas, revistas nacionales indexadas, y revistas especializadas nacionales e internacionales (factor 26, indicadores 70-72). La indexación aquí se refiere a la base de datos ScienTI y las bases de datos internacionales seleccionadas por Colciencias.

Ahora, dadas estas políticas, interesa ver cómo se reflejan en los resultados de la producción científica colombiana registrada en la base de datos Scopus, durante el período 2003-2011 y en otros índices con visibilidad iberoamericana. 
Tabla 1. Revistas indexadas en Publindex según tipo de institución editora, 2002-2011

\begin{tabular}{|c|c|c|c|c|c|c|c|c|c|c|c|c|c|c|}
\hline Institución editora & 2002 & 2003 & 2004 & 2005 & 2006 & 2007 & 2008 & 2009 & 2010 & \multicolumn{5}{|c|}{2011} \\
\hline & & & & & & & & & & $\mathrm{A} 1$ & $\mathrm{~A} 2$ & $\mathrm{~B}$ & $\mathrm{C}$ & Total \\
\hline IES privadas & 26 & 16 & 38 & 48 & 65 & 82 & 112 & 144 & 184 & 7 & 45 & 46 & 138 & 236 \\
\hline IES públicas & 47 & 36 & 58 & 75 & 99 & 105 & 114 & 125 & 148 & 13 & 36 & 39 & 89 & 177 \\
\hline $\begin{array}{l}\text { ONGs, asociaciones y } \\
\text { agremiaciones profesionales }\end{array}$ & 12 & 7 & 14 & 19 & 23 & 21 & 21 & 22 & 23 & 4 & 8 & 8 & 11 & 31 \\
\hline $\begin{array}{l}\text { Centros de investigación y } \\
\text { desarrollo tecnológico }\end{array}$ & 2 & 2 & 6 & 8 & 8 & 7 & 7 & 7 & 9 & 0 & 2 & 1 & 8 & 11 \\
\hline Entidades gubernamentales & 3 & 1 & 2 & 2 & 2 & 2 & 3 & 5 & 4 & 1 & 0 & 4 & 3 & 8 \\
\hline Hospitales y clínicas & 0 & 0 & 0 & 1 & 2 & 2 & 2 & 2 & 1 & 0 & 0 & 0 & 1 & 1 \\
\hline Empresas públicas, privadas y mixtas & 1 & 0 & 1 & 2 & 2 & 2 & 2 & 2 & 2 & 0 & 2 & 0 & 0 & 2 \\
\hline $\begin{array}{l}\text { Instituciones privadas sin fines de lucro } \\
\text { al servicio de las empresas }\end{array}$ & 0 & 0 & 0 & 1 & 1 & 1 & 2 & 1 & 1 & 0 & 0 & 1 & 0 & 1 \\
\hline Total & 91 & 62 & 119 & 156 & 202 & 222 & 263 & 308 & 372 & 25 & 93 & 99 & 250 & $467^{*}$ \\
\hline
\end{tabular}

* La suma es mayor que el número de revistas indexadas, porque una revista es publicada por dos instituciones.

Tomado de: Colombia. Colciencias (2012)

\section{Material y método}

Se trata de un estudio descriptivo y comparativo llevado a cabo en el contexto del análisis del dominio científico colombiano a partir de datos Scopus. Por tanto, la información sobre las revistas y producción científica de Colombia se obtuvo de la plataforma SCImago Journal and Country Rank (SCImago Research Group, 2013) de donde también se tomaron los datos correspondientes a los países latinoamericanos con mayor producción: Brasil, México, Argentina y Chile. En la misma línea, se consultaron las plataformas Web of science, Redalyc y SciELO.

La información sobre Publindex se obtuvo directamente del portal de Colciencias, y de los documentos soporte que el mismo sitio ofrece. Los documentos de política se recuperaron directamente de sitios web gubernamentales.

\section{Resultados y discusión}

Para empezar, hay que resaltar el hecho de que hablar de "revistas indexadas" en Colombia, es hablar del índice Publindex, lo que también contrasta con la costumbre del mundo de la ciencia, y ha determinado una mirada nacional más que internacional sobre la publicación científica. Este índice refleja los resultados de las políticas enunciadas, que a su vez se basan en los criterios establecidos por este sistema para la conformación del Índice Bibliográfico Nacional.

De acuerdo con los indicadores que revela el mismo Publindex (Colombia. Colciencias, 2012), en 2011 se registraban 466 títulos, de los cuales el $5 \%$ se clasifica en la categoría A1; el $20 \%$ en A2; el $21 \%$ en B y $54 \%$ en C. Pero lo revelador está en la tabla 1 , que muestra cómo las principales entidades editoras son las instituciones de educación superior (IES), que sumadas representan un $88 \%$ del conjunto. El mayor peso lo tienen las IES privadas, con prácticamente la mitad (el 51\%).

En esta proliferación de revistas científicas en las IES, incide la política del SUE que incluye un indicador referido a las revistas de la institución indexadas en Publindex. Sin embargo, no es claro el comportamiento que tienen las IES privadas. Como se observa en la tabla, la tendencia es siempre creciente, al punto de tener un total de 466 títulos en 2011, cuando en el 2002 se registraban 91. Esta situación, a su vez no se corresponde con las capacidades de investigación del país, donde apenas se empieza a reaccionar a las tendencias internacionales en materia de ciencia, tecnología e innovación, y tampoco cuenta con el nivel de profesionalización que exige cualquier proceso de edición de revistas científicas.

El mismo Publindex reconoce, que "si bien a 10 años de creado el servicio hay un importante crecimiento de la producción científica nacional, también lo es que la mayoría de las publicaciones integradas en el IBN Publindex carecen de poca

Figura 1. Evolución de las revistas latinoamericanas en Scopus, 1999-2012

Fuente: SCImago Research Group a partir de datos Scopus. 
visibilidad e impacto en la comunidad internacional y son de orden endogámico, evidenciado en el bajo porcentaje de revistas nacionales integradas en índices de citación que miden impacto. Actualmente el $4,77 \%$ (22 revistas) cuentan con factor de impacto y el $53,6 \%$ (250 revistas) que están en categoría $\mathrm{C}$ son de carácter endógeno" (Colombia. Colciencias, 2013a, p. 2)

Igual consideración hacen

Romero-Torres, AcostaMoreno y Tejada-Gómez (2013) cuando dicen que es necesario abandonar el actual sistema de clasificación basado en categorías

A1, A2, B y C, para migrar a un sistema de clasificación que utilice indicadores que faciliten la homologación y comparación de revistas internacionalmente. Para ello proponen un método basado en el índice $h$, aunque el modelo lanzado en 2013 por Colciencias todavía no lo considera; en cambio, sí coincide en la idea de reclasificar las categorías de indexación.

Para ofrecer un contexto, la figura 1 muestra la evolución de las revistas latinoamericanas en Scopus, que mantiene una tendencia positiva entre 1999 y 2012. En total, son 590 títulos, de los cuales el $10 \%$ son colombianos. Los 590 títulos latinoamericanos contienen 105.021 documentos publicados en 2012, muchos de los cuales son también producción nacional, como se observa en la figura 2.

La mayor tasa de endogamia la presenta Brasil, que excede en mucho el promedio latinoamericano, a la vez que lo eleva. Colombia se encuentra dentro de ese promedio, pero debería ubicarse por debajo del $20 \%$, como bien lo hacen México y Argentina. La tasa de endogamia colombiana claramente refleja las políticas nacionales que favorecen la publicación de producción científica en las revistas colombianas indexadas en Publindex.

La figura 3 muestra las revistas nacionales presentes en las bases de datos Scopus, Web of science, Redalyc y SciELO de los mismos países latinoamericanos. Gracias a ella se ob- serva la apuesta que hace Colombia por las bases de datos regionales SciELO y Redalyc, mucho más accesibles que Web of science y Scopus. Si bien son logros importantes para una cultura que apenas empieza a incursionar en la comunicación científica con visibilidad internacional, es claro que los países vecinos aventajan a Colombia, excepto Argentina.

\section{Conclusiones}

Las políticas que incentivan la comunicación científica colombiana varían conforme se avanza en las capacidades nacionales para la ciencia, la tecnología y la innovación, y el país se pone a tono con las tendencias mundiales del sector.

Todos los incentivos para la producción científica de los investigadores están determinados por el Sistema Nacional de Indexación y Homologación de Revistas Especializadas en $C T+l$ Publindex, creado por Colciencias, en tanto que para

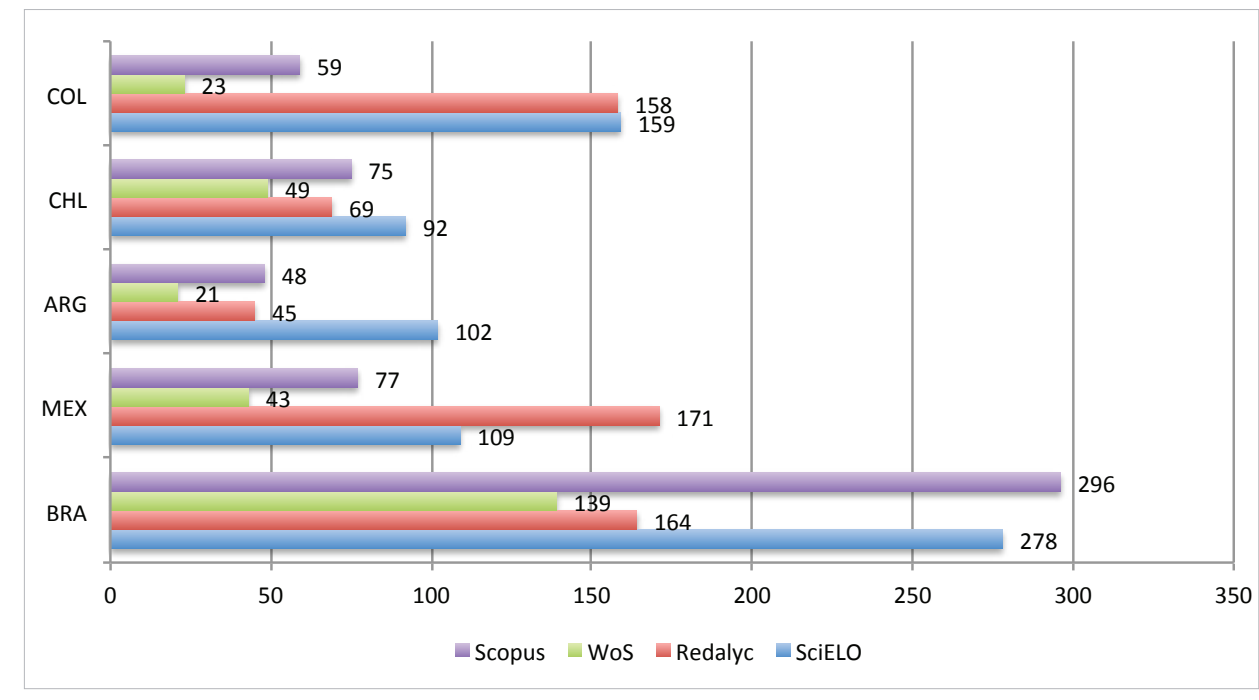

Figura 3. Revistas nacionales en bases de datos con visibilidad internacional, 2012 y 2013.

Fuente: SCImago Research Group (2013) a partir de datos Scopus; Thomson Reuters (2013); Redalyc (2013); SCIELO (2013) 
las instituciones de educación superior se tiene en cuenta, además, el número de revistas indexadas en Publindex que ellas editan.

La estructura de este Sistema ha tenido dos efectos adversos: una valoración de revistas nacionales que no se corresponde con criterios internacionales; y la edición de un número importante de títulos propios que no se corresponde con la capacidad científica del país. En consecuencia, este Sistema ha hecho que no se traten en igualdad de condiciones todas las publicaciones y por tanto, no constituya un sistema eficiente de incentivos. Para corregirlo, es necesario estimular la publicación científica de los investigadores en función del nivel de las revistas y no de su nacionalidad.

Como efectos positivos, se pueden mencionar dos: una mayor cultura de la comunicación científica entre los actores del Sistema Nacional de Ciencia, Tecnología e Innovación; y unas mejores prácticas en la edición científica que han permitido el ingreso de revistas colombianas en los índices internacionales de citaciones. Esta situación favorable coincide con la política de ampliación de cobertura geográfica de Scopus, que al aplicarse, encuentra publicaciones colombianas que se ajustan a sus criterios de calidad internacional. Ambos efectos han permitido una mayor presencia de Colombia en las bases de datos de citaciones, con un destacado incremento en la producción científica respecto a sus vecinos latinoamericanos. No obstante, las revistas colombianas deben moderar sus tasas de endogamia e incrementar la internacionalización para mejorar sus indicadores de impacto y su respectiva ubicación por cuartiles. En este propósito, debe cambiarse el Decreto 1279 cuando restringe el número de autores por artículo.

El panorama descrito está en proceso de cambio, pues en 2013 se producen unos nuevos lineamientos de Publindex tanto para la indexación de revistas como para la clasificación de los sires, con la intención de moverse con mayor decisión hacia el contexto internacional. A la vez, está en proceso de modificación el Decreto 1279 que también busca cambios sustanciales en el sistema de incentivos para los profesores universitarios.

\section{Referencias}

Charum, Jorge (2004). "La construcción de un sistema nacional de indexación, el caso de Publindex". Convergencia, mayo-agosto, v. 11, n. 35, pp. 293-309.

Colombia (1992a). Ley 30 de diciembre 28 de 1992: por el cual se organiza el servicio público de la educación superior. Bogotá: Congreso de la República. 26 pp.

http://www.mineducacion.gov.co/1621/articles-86437 Archivo_pdf.pdf

Colombia (1992b). Decreto 1444 de 1992. Bogotá: Presidencia de la República.

http://www.mineducacion.gov.co/1621/articles-103567_ archivo_pdf.pdf

Colombia (2001). Decreto 2912 del 31 de diciembre de 2001. Bogotá: Presidencia de la República. 39 pp.

http://www.mineducacion.gov.co/1621/articles-86123_ archivo_pdf.pdf
Colombia (2002). Decreto 1279 de junio 19 de 2002. Bogotá: Presidencia de la República. 30 pp. http://www.mineducacion.gov.co/1621/articles-86434_ Archivo_pdf.pdf

Colombia. Colciencias (2012). Indicadores generales Publindex 2002-2011. Bogotá: Colciencias. 8 pp.

http://www.colciencias.gov.co/sites/default/files/ ckeditor_files/files/INDICADORES\%20GENERALES\%20 PUBLINDEX\%202011.pdf

Colombia. Colciencias (2013a). Revistas especializadas de CTI. http://www.colciencias.gov.co/programa_estrategia/ revistas-especializadas-de-cti- 0

Colombia. Colciencias (2013b). Documento guía para el Servicio Permanente de Indexación de Revistas Colombianas de Ciencia, Tecnología e Innovación, 2013. Bogotá: Colciencias. $15 \mathrm{pp}$.

Colombia. Colciencias (2013c). Servicios de indexación y resumen - SIR utilizados para los procesos de indexación y homologación de revistas especializadas de CTel. Bogotá: Colciencias. 15 pp.

http://www.colciencias.gov.co/sites/default/files/ckeditor_ files/files/Documento\%20SIRES\%20-\%202013.pdf

Colombia. Colciencias (2013d). Modelo de medición de grupos de investigación, desarrollo tecnológico y/o de innovación. Bogotá: Colciencias. 126 pp.

http://www.colciencias.gov.co/sites/default/files/upload/ documents/modelo_de_medicion_grupos_2013-definitiva.pdf

Colombia. Consejo Nacional de Acreditación (2010). Autoevaluación con fines de acreditación de alta calidad de programas de maestría y doctorado: guía de procedimiento. Bogotá: CNA. 49 pp.

http://cms-static.colombiaaprende.edu.co/cache/binaries/ articles-186363_lineam_MyD.pdf?binary_rand $=7259$

Colombia. Consejo Nacional de Acreditación (2006). Indicadores para la autoevaluación con fines de acreditación institucional. 2ª ed. Bogotá: CNA, Ascun. 61 pp.

http://cms-static.colombiaaprende.edu.co/cache/binaries/ articles-186376_indi_acreinstit.pdf?binary_rand $=4489$

Colombia. Ministerio de Educación Nacional (2012). Propuesta metodológica para la distribución de recursos artículo 87 de la Ley 30 de 1992, vigencia 2012. Bogotá: Ministerio de Educación Nacional. 6 pp.

ht t p://ww w. min educacion.gov.co/ sistemasdeinformacion/1735/articles-212353_modelo1.pdf

Colombia. Sistema Universitario Estatal (2012). Desfinanciamiento de la educación superior en Colombia. Bogotá: SUE. $58 \mathrm{pp}$.

http://www.almamater.edu.co/sitio/Archivos/Documentos/ Documentos/00000810.pdf

Delgado-Troncoso, Jorge-Enrique (2009). Advances and challenges in scientific journal publication in Colombia: analysis of institutions and publications. Pittsburgh: University of Pittsburgh. 13 pp., working paper; March 9.

http://www.academia.edu/192067/Advances_and_ challenges_in_scientific_journal_publication_in_ 
Colombia_-_Analysis_of_institutions_and_publications

Redalyc (2013). Red de Revistas Cientificas de América Latina y el Caribe, España y Portugal: sistema de información científica. http://www.redalyc.org

Romero-Torres, Mauricio; Acosta-Moreno, Luis-Alberto; Tejada-Gómez, María-Alejandra (2013). “Ranking de revistas científicas en Latinoamérica mediante el índice h: estudio de caso Colombia". Revista española de documentación científica, enero-marzo, v. 36, n. 1, pp. 1-13 http://dx.doi.org/10.3989/redc.2013.1.876

SciELO (2013). Scientific Electronic Library Online.

http://www.scielo.org/php/index.php?lang=es

SCImago Research Group (2013). SCImago Journal and Country Rank.

http://www.scimagojr.com

Thomson Reuters (2013). Master journal list

http://ip-science.thomsonreuters.com/mjl

\section{Colección de libros de bolsillo}

\section{El profesional de la información (Editorial UOC)}
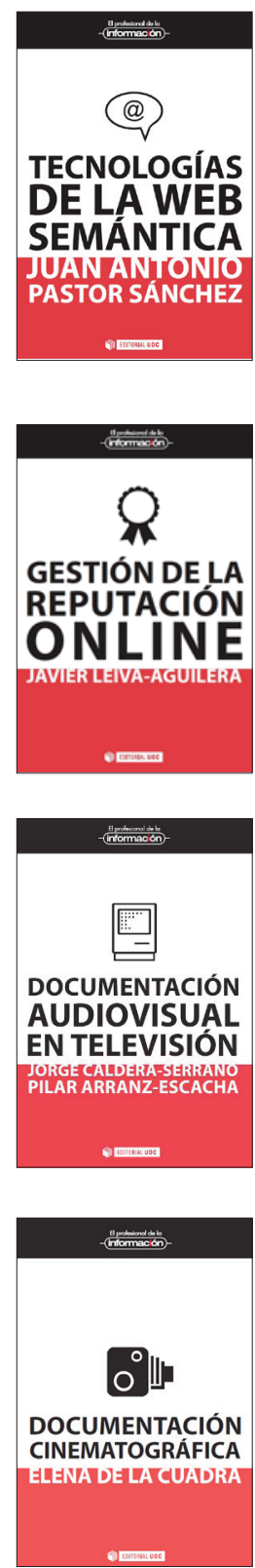
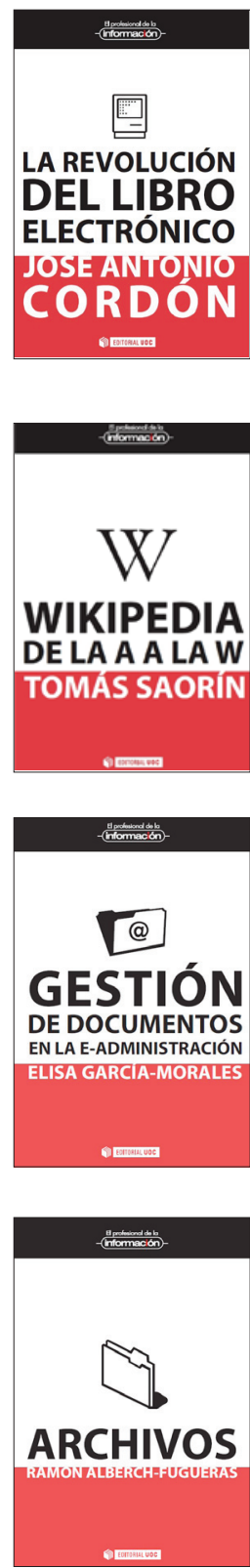
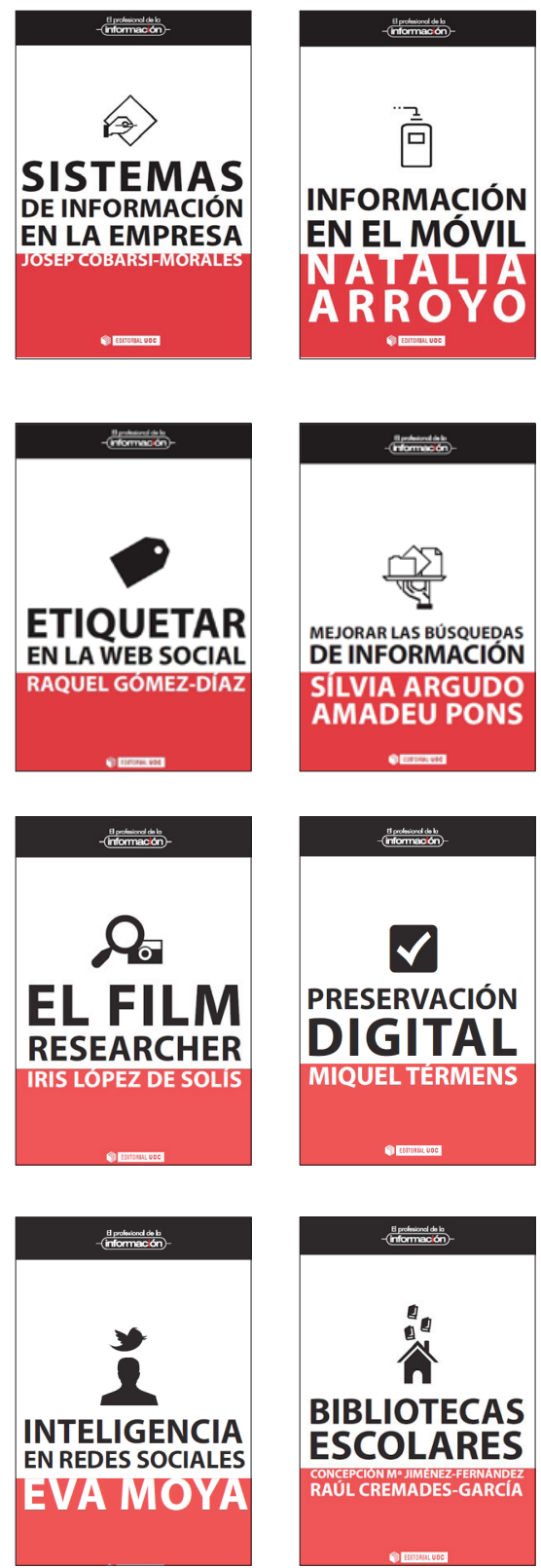
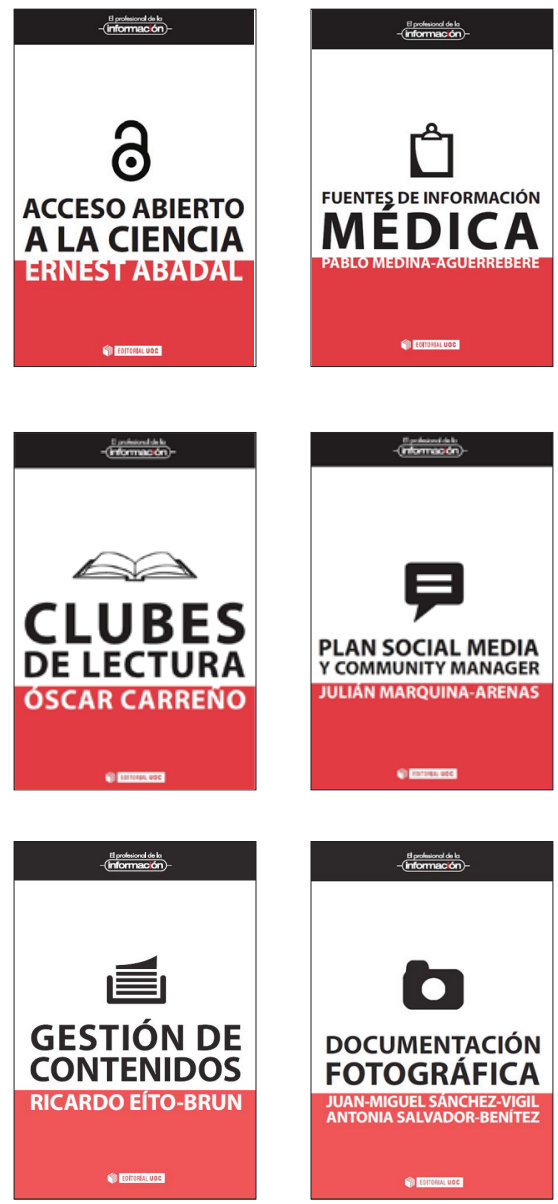

\section{Más información:}


